\title{
ON THE GLOBAL WELL-POSEDNESS OF THE CRITICAL QUASI-GEOSTROPHIC EQUATION
}

\author{
HAMMADI ABIDI AND TAOUFIK HMIDI
}

\begin{abstract}
We prove the global well-posedness of the critical dissipative quasi-geostrophic equation for large initial data belonging to the critical Besov space $\dot{B}_{\infty, 1}^{0}\left(\mathbb{R}^{2}\right)$.
\end{abstract}

\section{INTRODUCTION}

In this paper we are concerned with the initial value problem of the $2 \mathrm{D}$ dissipative quasi-geostrophic equation

$$
(\mathrm{QG})_{\alpha}\left\{\begin{array}{l}
\partial_{t} \theta+v \cdot \nabla \theta+|\mathrm{D}|^{\alpha} \theta=0 \\
\theta_{\mid t=0}=\theta^{0}
\end{array}\right.
$$

where the scalar function $\theta$ represents the potential temperature and the parameter $\alpha \in] 0,2]$. The velocity $v=\left(v^{1}, v^{2}\right)$ is determined by Riesz transforms of $\theta$ :

$$
v=\left(-\partial_{2}|\mathrm{D}|^{-1} \theta, \partial_{1}|\mathrm{D}|^{-1} \theta\right):=\left(-R_{2} \theta, R_{1} \theta\right) ; \quad|\mathrm{D}|=\sqrt{-\Delta} .
$$

In addition to its intrinsic mathematical importance this equation serves as a 2D model in geophysical fluid dynamics, for more details about the subject see [6, 19].

This equation has been intensively investigated and much attention is carried to the problem of global well-posedness. For the sub-critical case $(\alpha>1)$ the theory seems to be in a satisfactory state. Indeed, global existence and uniqueness for arbitrary initial data are established in various function spaces (see for example [8, 20]). However the critical and super-critical cases, corresponding respectively to $\alpha=1$ and $\alpha<1$, are harder to deal with. In the super-critical case, we have until now only global results for small initial data, see for instance [3, 5, 12, 13, 23, 24]. For critical case , Constantin, Córdoba and $\mathrm{Wu}$ showed in [7] the global existence in Sobolev space $H^{1}$ under smallness assumption of $L^{\infty}$ norm of $\theta^{0}$. Many other relevant results can be found in [9, 14, 15, 17]. Very recently, Kiselev, Nazarov and Volberg proved in [16] the global well-posedness for arbitrary periodic smooth initial data by using an elegant argument of modulus of continuity. In [2], Caffarelli and Vasseur established the global regularity of weak solutions associated to $L^{2}$ initial data.

The main goal of this work is to establish the global well-posedness in the critical case when initial data belong to the homogeneous critical Besov 
space $\dot{B}_{\infty, 1}^{0}\left(\mathbb{R}^{2}\right)$ : we remove the periodic condition and we weaken the initial regularity. Before giving our main result let us first precise the notion of critical spaces. Let $\theta$ be a solution of $(\mathrm{QG})_{\frac{1}{2}}$ and $\lambda>0$ then $\theta_{\lambda}(t, x)=$ $\theta(\lambda t, \lambda x)$ is also a solution. One class of scaling invariant spaces is the homogeneous Besov spaces $\left(\dot{B}_{p, r}^{\frac{2}{p}}\right)$, with $p, r \in[1, \infty]$.

Our first main result reads as follows.

Theorem 1.1. Let $\theta^{0} \in \dot{B}_{\infty, 1}^{0}$, then there exists a unique global solution $\theta$ to $(\mathrm{QG})_{\frac{1}{2}}$ such that

$$
\theta \in \mathcal{C}\left(\mathbb{R}_{+} ; \dot{B}_{\infty, 1}^{0}\right) \cap L_{\mathrm{loc}}^{1}\left(\mathbb{R}_{+} ; \dot{B}_{\infty, 1}^{1}\right) .
$$

The proof relies essentially on two facts: the first one is the establishement of the local existence which is the major part of this paper and the derivation of some smoothing effects of the solution, described in next Theorem. The second one is the use of the modulus of continuity as done in [16]. We mention that the property allowing us to remove the periodicity is the spatial decay of the solution. The key of our local existence result is some new estimates for the following transport-diffusion equation :

$$
(\mathrm{TD})\left\{\begin{array}{l}
\partial_{t} \theta+v \cdot \nabla \theta+|\mathrm{D}| \theta=f \\
\theta_{\mid t=0}=\theta^{0}
\end{array}\right.
$$

where $v$ and $f$ are given and $\theta$ is the unknown scalar function. We state now our second main result.

Theorem 1.2. Let $s \in]-1,1[, r, \bar{r} \in[1,+\infty]$ with $r \geq \bar{r}, f \in$ $\widetilde{L}_{\text {loc }}^{\bar{r}}\left(\mathbb{R}_{+} ; \dot{B}_{\infty, 1}^{s+\frac{1}{\bar{r}}-1}\right)$ and $v$ be a divergence free vector field belonging to $L_{\text {loc }}^{1}\left(\mathbb{R}_{+} ; \operatorname{Lip}\left(\mathbb{R}^{2}\right)\right)$. We consider a smooth solution $\theta$ of the transportdiffusion equation (TD), then there exists a constant $C$ depending only on $s$ such that for every $t \in \mathbb{R}_{+}$

$$
\|\theta\|_{\widetilde{L}_{t}^{r} \dot{B}_{\infty, 1}^{s+\frac{1}{r}}} \leq C e^{C \int_{0}^{t}\|\nabla v(\tau)\|_{L} \infty d \tau}\left(\left\|\theta^{0}\right\|_{\dot{B}_{\infty, 1}^{s}}+\|f\|_{\widetilde{L}_{t}^{r} \dot{B}_{\infty, 1}^{s+\frac{1}{r}-1}}\right) .
$$

Besides if $v=\nabla^{\perp}|\mathrm{D}|^{-1} \theta$, then we have for all $s \geq 1$

$$
\|\theta\|_{\widetilde{L}_{t}^{r} \dot{B}_{\infty, 1}^{s+\frac{1}{r}}} \leq C e^{C \int_{0}^{t}\left(\|\nabla \theta(\tau)\|_{L}+\|\nabla v(\tau)\|_{L} \infty\right) d \tau}\left(\left\|\theta^{0}\right\|_{\dot{B}_{\infty, 1}^{s}}+\|f\|_{\widetilde{L}_{t}^{r} \dot{B}_{\infty, 1}^{s+\frac{1}{r}-1}}\right) .
$$

We use for the proof a new approach based on Lagrangian coordinates combined with paradifferential calculus and a new commutator estimate. This idea has been recently used by the second author in [11, 12].

The rest of the paper is structured as follows. In section 2 we review some basic results of Littlewood-Paley theory and we give some useful lemmas. Section 3 deals with a new commutator estimate which is needed for the proof of Theorem 1.2, done in section 4. Theorem 1.1 is proved in section 5 . 


\section{Preliminaries}

Throughout the paper, $C$ stands for a constant which may be different in each occurrence. We shall sometimes use the notation $A \lesssim B$ instead of $A \leq C B$ and $A \approx B$ means that $A \lesssim B$ and $B \lesssim A$. We denote by $\mathcal{F} f$ the Fourier transform of $f$ and $[\eta]$ by the whole part of $\eta$.

One starts with recalling a traditional result that will be frequently used.

Lemma 2.1. (Bernstein) Let $f \in L^{p}\left(\mathbb{R}^{2}\right)$, with $1 \leq p \leq \infty$ and $0<r<R$. Then there exists a constant $C>0$ such that $\forall k \in \mathbb{N}$ and $\lambda>0$, we have:

$$
\begin{aligned}
& \text { supp } \mathcal{F} f \subset B(0, \lambda r) \Longrightarrow \sup _{|\beta|=k}\left\|\partial^{\beta} f\right\|_{L^{q}} \leq C^{k} \lambda^{k+2\left(\frac{1}{p}-\frac{1}{q}\right)}\|f\|_{L^{p}} \\
& \operatorname{supp} \mathcal{F} f \subset \mathcal{C}(0, \lambda r, \lambda R) \Longrightarrow C^{-k} \lambda^{k}\|f\|_{L^{p}} \leq \sup _{|\beta|=k}\left\|\partial^{\beta} f\right\|_{L^{p}} \leq C^{k} \lambda^{k}\|f\|_{L^{p}} .
\end{aligned}
$$

These estimates hold true if we replace the derivation $\partial^{\beta}$ by $|\mathrm{D}|^{|\beta|}$.

To define Besov spaces we need to recall the homogeneous Littlewood-Paley decomposition based on a dyadic unity partition. Let $\varphi$ be a smooth function supported in the $\operatorname{ring} \mathcal{C}:=\left\{\xi \in \mathbb{R}^{2}, \frac{3}{4} \leq|\xi| \leq \frac{8}{3}\right\}$ and such that

$$
\sum_{q \in \mathbb{Z}} \varphi\left(2^{-q} \xi\right)=1 \quad \text { for } \quad \xi \neq 0 .
$$

Now, for $u \in \mathcal{S}^{\prime}$ we set

$$
\forall q \in \mathbb{Z}, \quad \Delta_{q} u=\varphi\left(2^{-q} \mathrm{D}\right) u \quad \text { and } \quad S_{q} u=\sum_{j \leq q-1} \Delta_{j} u .
$$

We have the formal decomposition

$$
u=\sum_{q \in \mathbb{Z}} \Delta_{q} u, \quad \forall u \in \mathcal{S}^{\prime}\left(\mathbb{R}^{2}\right) / \mathcal{P}\left[\mathbb{R}^{2}\right],
$$

where $\mathcal{P}\left[\mathbb{R}^{2}\right]$ is the set of polynomials (see [18]). Moreover, the LittlewoodPaley decomposition satisfies the property of almost orthogonality:

$$
\Delta_{k} \Delta_{q} u \equiv 0 \quad \text { if } \quad|k-q| \geq 2 \quad \text { and } \quad \Delta_{k}\left(S_{q-1} u \Delta_{q} u\right) \equiv 0 \quad \text { if } \quad|k-q| \geq 5 .
$$

We recall now the definition of Besov spaces. Let $(p, m) \in[1,+\infty]^{2}, s \in \mathbb{R}$ and $u \in \mathcal{S}^{\prime}$, we set

$$
\|u\|_{\dot{B}_{p, m}^{s}}:=\left(2^{q s}\left\|\Delta_{q} u\right\|_{L^{p}}\right)_{\ell^{m}} ; \quad \dot{\mathcal{B}}_{p, m}^{s}:=\left\{u \in \mathcal{S} \mid\|u\|_{\dot{B}_{p, m}^{s}}<\infty\right\} .
$$

- For $s<\frac{2}{p}$ (or $s \leq \frac{2}{p}$ if $m=1$ ), we then define $\dot{B}_{p, m}^{s}$ as the completion of $\dot{\mathcal{B}}_{p, m}^{s}$ for $\|\cdot\|_{\dot{B}_{p, m}^{s}}$.

- If $k \in \mathbb{N}$ and $\frac{2}{p}+k-1 \leq s<\frac{2}{p}+k$ (or $s=\frac{2}{p}+k$ if $m=1$ ), then $\dot{B}_{p, m}^{s}$ is defined as the subset of distributions $u \in \mathcal{S}^{\prime}$ such that $\partial^{\beta} u \in \dot{B}_{p, m}^{s-k}$ whenever $|\beta|=k$. 
Another characterization of the homogeneous Besov spaces that will be needed later is the following, see for instance [21. For $s \in] 0,1[, p, m \in[1, \infty]$

$$
\left(\int_{\mathbb{R}^{2}} \frac{\|u(\cdot-x)-u(\cdot)\|_{L^{p}}^{m}}{|x|^{s m}} \frac{d x}{|x|^{2}}\right)^{\frac{1}{m}} \approx\|u\|_{\dot{B}_{p, m}^{s}},
$$

with the usual modification if $m=\infty$.

In our next study we require two kinds of coupled space-time Besov spaces. The first one is defined by the following manner: for $T>0$ and $m \geq 1$, we denote by $L_{T}^{r} \dot{B}_{p, m}^{s}$ the set of all tempered distribution $u$ satisfying

$$
\|u\|_{L_{T}^{r} \dot{B}_{p, m}^{s}}:=\left\|\left(2^{q s}\left\|\Delta_{q} u\right\|_{L^{p}}\right)_{\ell^{m}}\right\|_{L_{T}^{r}}<\infty .
$$

The second mixed space is $\widetilde{L}_{T}^{r} \dot{B}_{p, m}^{s}$ which is the set of tempered distribution $u$ satisfying

$$
\|u\|_{\widetilde{L}_{T}^{r} \dot{B}_{p, m}^{s}}:=\left(2^{q s}\left\|\Delta_{q} u\right\|_{L_{T}^{r} L^{p}}\right)_{\ell^{m}}<\infty .
$$

We can define by the same way the spaces $L_{T}^{r} B_{p, m}^{s}$ and $\widetilde{L}_{T}^{r} B_{p, m}^{s}$. The following embeddings are a direct consequence of Minkowski's inequality.

Let $s \in \mathbb{R}, r \geq 1$ and $(p, m) \in[1, \infty]^{2}$, then we have

$$
\begin{aligned}
& L_{T}^{r} \dot{B}_{p, m}^{s} \hookrightarrow \widetilde{L}_{T}^{r} \dot{B}_{p, m}^{s} \text {, if } m \geq r \quad \text { and } \\
& \widetilde{L}_{T}^{r} \dot{B}_{p, m}^{s} \hookrightarrow L_{T}^{r} \dot{B}_{p, m}^{s} \text {, if } \quad r \geq m .
\end{aligned}
$$

The next lemma will be useful.

Proposition 2.2. The following results hold true:

$$
\dot{B}_{p, m}^{s} \hookrightarrow \dot{B}_{p_{1}, m_{1}}^{s-2\left(\frac{1}{p}-\frac{1}{p_{1}}\right)}, \quad \text { for } \quad p \leq p_{1} \quad \text { and } \quad m \leq m_{1} .
$$

- Let $|\mathrm{D}|:=\sqrt{-\Delta}$ and $\sigma \in \mathbb{R}$, then the operator $|\mathrm{D}|^{\sigma}$ is an isomorphism from $\dot{B}_{p, m}^{s}$ to $\dot{B}_{p, m}^{s-\sigma}$.

- Let $\gamma \in] 0,1\left[, s_{1}, s_{2} \in \mathbb{R}\right.$ such that $s_{1}<s_{2}$ and $u \in \dot{B}_{p, \infty}^{s_{1}} \cap \dot{B}_{p, \infty}^{s_{2}}$, then

$$
\|u\|_{\dot{B}_{p, 1}^{\gamma s_{1}+(1-\gamma) s_{2}}} \lesssim\|u\|_{\dot{B}_{p, \infty}^{s_{1}}}^{\gamma}\|u\|_{\dot{B}_{p, \infty}^{s_{2}}}^{1-\gamma} .
$$

- For $s>0, \dot{B}_{p, m}^{s} \cap L^{\infty}$ is an algebra.

We now recall some commutator estimates (see [4, 10] and the references therein).

Lemma 2.3. Let $p, r \in[1, \infty], 1=\frac{1}{r}+\frac{1}{r^{\prime}}, \rho_{1}<1, \rho_{2}<1$ and $v$ be a divergence free vector field of $\mathbb{R}^{2}$. Assume in addition that

$$
\rho_{1}+\rho_{2}+2 \min \{1,2 / p\}>0 \text { and } \rho_{1}+2 / p>0 .
$$

Then we have

$$
\sum_{q \in \mathbb{Z}} 2^{q\left(\frac{2}{p}+\rho_{1}+\rho_{2}-1\right)}\left\|\left[\Delta_{q}, v \cdot \nabla\right] u\right\|_{L_{t}^{1} L^{p}} \lesssim\|v\|_{\widetilde{L}_{t}^{r} \dot{B}_{p, 1}^{\frac{2}{p}+\rho_{1}}}\|u\|_{\widetilde{L}_{t}^{r^{\prime}} \dot{B}_{p, 1}^{\frac{2}{p}+\rho_{2}}} .
$$


Moreover we have for $s \in]-1,1[$

$$
\sum_{q \in \mathbb{Z}} 2^{q s}\left\|\left[\Delta_{q}, v \cdot \nabla\right] u\right\|_{L^{p}} \lesssim\|\nabla v\|_{L^{\infty}}\|u\|_{\dot{B}_{p, 1}^{s}} .
$$

If $v=\nabla^{\perp}|\mathrm{D}|^{-1} \theta$, then the above estimate holds true for $s \geq 1$ if we replace $\|\nabla v\|_{L^{\infty}}$ by $\|\nabla v\|_{L^{\infty}}+\|\nabla u\|_{L^{\infty}}$.

The following result is due to Vishik [22].

Lemma 2.4. Let $f$ be a function in Schwartz class and $\psi$ a diffeomorphism preserving Lebesgue measure, then for all $p \in[1,+\infty]$ and for all $j, q \in \mathbb{Z}$,

$$
\left\|\Delta_{j}\left(\Delta_{q} f \circ \psi\right)\right\|_{L^{p}} \leq C 2^{-|j-q|}\left\|\nabla \psi^{\epsilon(j, q)}\right\|_{L^{\infty}}\left\|\Delta_{q} f\right\|_{L^{p}}
$$

with

$$
\epsilon(j, q)=\operatorname{sign}(j-q) .
$$

The following result is proved in $[9]$.

Proposition 2.5. Let $v$ be a smooth divergence free vector field and $f$ be a smooth function. We assume that $\theta$ is a smooth solution of the equation

$$
\partial_{t} \theta+v \cdot \nabla \theta+\kappa|\mathrm{D}|^{\alpha} \theta=f, \quad \text { with } \quad \kappa \geq 0 \quad \text { and } \quad \alpha \in[0,2] .
$$

Then for $p \in[1,+\infty]$ we have

$$
\|\theta(t)\|_{L^{p}} \leq\|\theta(0)\|_{L^{p}}+\int_{0}^{t}\|f(\tau)\|_{L^{p}} d \tau
$$

We can find a proof of the next proposition in [12].

Proposition 2.6. Let $\mathcal{C}$ be a ring and $\alpha \in \mathbb{R}_{+}$. There exists a positive constant $C$ such that for any $p \in[1 ;+\infty]$, for any couple $(t, \lambda)$ of positive real numbers, we have

$$
\operatorname{supp} \mathcal{F} u \subset \lambda \mathcal{C} \Rightarrow\left\|e^{-t|\mathrm{D}|^{\alpha}} u\right\|_{L^{p}} \leq C e^{-C^{-1} t \lambda^{\alpha}}\|u\|_{L^{p}}
$$

\section{Commutator estimate}

The main result of this section is the following estimate that will play a crucial role for the proof of Theorem 1.2 .

Proposition 3.1. Let $v$ be a divergence free vector field belonging to $L_{\text {loc }}^{1}\left(\mathbb{R}_{+} ; \operatorname{Lip}\left(\mathbb{R}^{2}\right)\right)$. For $q \in \mathbb{Z}$ we denote by $\psi_{q}$ the flow of the regularized vector field $S_{q-1} v$. Then for $f \in \dot{B}_{\infty, \infty}^{1}$ and for $q \in \mathbb{Z}$ we have

$$
\left\||\mathrm{D}|\left(\Delta_{q} f \circ \psi_{q}\right)-\left(|\mathrm{D}| \Delta_{q} f\right) \circ \psi_{q}\right\|_{L^{\infty}} \leq C e^{C V(t)} V^{\frac{1}{2}}(t) 2^{q}\left\|\Delta_{q} f\right\|_{L^{\infty}},
$$

where $V(t)=\|\nabla v\|_{L_{t}^{1} L^{\infty}\left(\mathbb{R}^{2}\right)}$ and $C$ an absolute constant. 
Proof. We set $f_{q}:=\Delta_{q} f$, then it is obvious that

$$
\begin{aligned}
|\mathrm{D}|\left(f_{q} \circ \psi_{q}\right)-\left(|\mathrm{D}| f_{q}\right) \circ \psi_{q} & =|\mathrm{D}|^{\frac{1}{2}}\left\{\left(|\mathrm{D}|^{\frac{1}{2}} f_{q}\right) \circ \psi_{q}\right\}-\left\{|\mathrm{D}|^{\frac{1}{2}}\left(|\mathrm{D}|^{\frac{1}{2}} f_{q}\right)\right\} \circ \psi_{q} \\
& +|\mathrm{D}|^{\frac{1}{2}}\left\{|\mathrm{D}|^{\frac{1}{2}}\left(f_{q} \circ \psi_{q}\right)-\left(|\mathrm{D}|^{\frac{1}{2}} f_{q}\right) \circ \psi_{q}\right\} \\
& :=\mathrm{I}+\mathrm{II} .
\end{aligned}
$$

For the first term we apply Proposition 3.1 [12], with $\alpha=\frac{1}{2}$ and $F_{q}=|\mathrm{D}|^{\frac{1}{2}} f_{q}$, yielding

$$
\begin{aligned}
\|\mathrm{I}\|_{L^{\infty}} & \leq C e^{C V(t)}\left(e^{C V(t)}-1\right)\left\|F_{q}\right\|_{\dot{B}_{\infty, 1}^{\frac{1}{2}}} \\
& \leq C e^{C V(t)}\left(e^{C V(t)}-1\right) 2^{q}\left\|f_{q}\right\|_{L^{\infty}} \\
& \lesssim e^{C V(t)} V^{\frac{1}{2}}(t) 2^{q}\left\|f_{q}\right\|_{L^{\infty}} .
\end{aligned}
$$

For the second term we use the following formula for the fractional Laplacian

$$
|\mathrm{D}|^{\frac{1}{2}} f(x)=C \int_{\mathbb{R}^{2}} \frac{f(x)-f(y)}{|x-y|^{\frac{5}{2}}} d y .
$$

Since the flow $\psi_{q}$ preserves Lebesgue measure then we get easily

$$
\begin{aligned}
|\mathrm{D}|^{\frac{1}{2}}\left(f_{q} \circ \psi_{q}\right)(x)-\left(|\mathrm{D}|^{\frac{1}{2}} f_{q}\right) \circ \psi_{q}(x)= & C \int_{\mathbb{R}^{2}} \frac{f_{q}\left(\psi_{q}(x)\right)-f_{q}\left(\psi_{q}(y)\right)}{|x-y|^{\frac{5}{2}}} \\
& \times\left(1-\frac{|x-y|^{\frac{5}{2}}}{\left|\psi_{q}(x)-\psi_{q}(y)\right|^{\frac{5}{2}}}\right) d y .
\end{aligned}
$$

We denote $g_{q}(x)=f_{q}\left(\psi_{q}(x)\right)$ and we put $h=x-y$ :

$$
|\mathrm{D}|^{\frac{1}{2}}\left(f_{q} \circ \psi_{q}\right)(x)-\left(|\mathrm{D}|^{\frac{1}{2}} f_{q}\right) \circ \psi_{q}(x)=C \int_{\mathbb{R}^{2}} \frac{g_{q}(x)-g_{q}(x-h)}{|h|^{\frac{5}{2}}} \bar{\psi}_{q}(x, h) d h,
$$

with

$$
\bar{\psi}_{q}(x, h)=1-\frac{|h|^{\frac{5}{2}}}{\left|\psi_{q}(x)-\psi_{q}(x-h)\right|^{\frac{5}{2}}} .
$$

It follows from law products and the embedding $\dot{B}_{\infty, 1}^{0} \hookrightarrow L^{\infty}$

$$
\begin{aligned}
\|\mathrm{II}\|_{L^{\infty}} & \leq\left\||\mathrm{D}|^{\frac{1}{2}}\left(f_{q} \circ \psi_{q}\right)-\left(|\mathrm{D}|^{\frac{1}{2}} f_{q}\right) \circ \psi_{q}\right\|_{\dot{B}_{\infty, 1}^{\frac{1}{2}}} \\
& \leq C\left\|\bar{\psi}_{q}\right\|_{L^{\infty}\left(\mathbb{R}^{4}\right)} \int_{\mathbb{R}^{2}}|h|^{-\frac{5}{2}}\left\|g_{q}(\cdot)-g_{q}(\cdot-h)\right\|_{\dot{B}_{\infty, 1}^{\frac{1}{2}}} d h \\
& +C \sup _{h \in \mathbb{R}^{2}}\left\|\bar{\psi}_{q}(\cdot, h)\right\|_{\dot{B}_{\infty, 1}^{\frac{1}{2}}} \int_{\mathbb{R}^{2}}|h|^{-\frac{5}{2}}\left\|g_{q}(\cdot)-g_{q}(\cdot-h)\right\|_{L^{\infty}} d h \\
& =J_{q}^{1}+J_{q}^{2} .
\end{aligned}
$$

We intend to estimate $J_{q}^{1}$. It is plain from Mean Value Theorem that

$$
\frac{1}{\|\nabla \psi\|_{L^{\infty}}^{\frac{5}{2}}} \leq \frac{|h|^{\frac{5}{2}}}{|\psi(x)-\psi(x-h)|^{\frac{5}{2}}} \leq\left\|\nabla \psi^{-1}\right\|_{L^{\infty}}^{\frac{5}{2}}
$$


which gives easily the inequality

$$
\left\|\bar{\psi}_{q}\right\|_{L^{\infty}\left(\mathbb{R}^{4}\right)} \leq \max \left(\left|1-\left\|\nabla \psi_{q}^{-1}\right\|_{L^{\infty}}^{\frac{5}{2}}\right| ;\left|1-\left\|\nabla \psi_{q}\right\|_{L^{\infty}}^{-\frac{5}{2}}\right|\right) .
$$

On the other hand we have the classical estimates

$$
\begin{array}{r}
e^{-C\left\|S_{q-1} \nabla v\right\|_{L_{t}^{1} L^{\infty}}} \leq\left\|\nabla \psi_{q}^{\mp 1}\right\|_{L^{\infty}} \leq e^{C\left\|S_{q-1} \nabla v\right\|_{L_{t}^{1} L^{\infty}}} \\
\text { and }\left\|S_{q-1} \nabla v\right\|_{L_{t}^{1} L^{\infty}} \leq C V(t) .
\end{array}
$$

We thus get

$$
\left\|\bar{\psi}_{q}\right\|_{L^{\infty}\left(\mathbb{R}^{4}\right)} \leq C e^{C V(t)}\left(e^{C V(t)}-1\right) .
$$

Using the definition of Besov spaces and the commutation of $\Delta_{j}$ with translation operators one finds

$$
\begin{aligned}
\int_{\mathbb{R}^{2}}|h|^{-\frac{5}{2}} \| g_{q}(\cdot) & -g_{q}(\cdot-h) \|_{\dot{B}_{\infty, 1}^{\frac{1}{2}}} d h \\
& \leq \sum_{j} 2^{\frac{1}{2} j} \int_{\mathbb{R}^{2}}|h|^{-\frac{1}{2}}\left\|\Delta_{j} g_{q}(\cdot)-\left(\Delta_{j} g_{q}\right)(\cdot-h)\right\|_{L^{\infty}} \frac{d h}{|h|^{2}} .
\end{aligned}
$$

Applying the characterization of Besov spaces (2) yields

$$
\begin{aligned}
\int_{\mathbb{R}^{2}}|h|^{-\frac{5}{2}}\left\|g_{q}(\cdot)-g_{q}(\cdot-h)\right\|_{\dot{B}_{\infty, 1}^{\frac{1}{2}}} d h & \leq C \sum_{j} 2^{\frac{1}{2} j}\left\|\Delta_{j} g_{q}\right\|_{\dot{B}_{\infty, 1}^{\frac{1}{2}}} \\
& \leq C \sum_{|j-k| \leq 1} 2^{j \frac{1}{2}} 2^{\frac{1}{2} k}\left\|\Delta_{j} \Delta_{k} g_{q}\right\|_{L^{\infty}} \\
& \leq C\left\|g_{q}\right\|_{\dot{B}_{\infty, 1}^{1}} .
\end{aligned}
$$

Now we use the following interpolation estimate

$$
\begin{aligned}
\left\|g_{q}\right\|_{\dot{B}_{\infty, 1}^{1}} & \lesssim\left\|g_{q}\right\|_{L^{\infty}}^{\frac{1}{2}}\left\|\Delta g_{q}\right\|_{L^{\infty}}^{\frac{1}{2}} \\
& \lesssim\left\|f_{q}\right\|_{L^{\infty}}^{\frac{1}{2}}\left\|\Delta g_{q}\right\|_{L^{\infty}}^{\frac{1}{2}} .
\end{aligned}
$$

It is easy to check from Leibnitz rule that

$$
\Delta g_{q}=\Delta\left(f_{q} \circ \psi_{q}\right)=\sum_{i=1}^{d}\left\langle\left(\nabla^{2} f_{q}\right) \circ \psi_{q} \cdot \partial_{i} \psi_{q}, \partial_{i} \psi_{q}\right\rangle+\left(\nabla f_{q}\right) \circ \psi_{q} \cdot \Delta \psi_{q} .
$$

Applying Bernstein inequality we get

$$
\left\|\Delta g_{q}\right\|_{L^{\infty}} \lesssim e^{C V(t)} 2^{2 q}\left\|f_{q}\right\|_{L^{\infty}}+2^{q}\left\|f_{q}\right\|_{L^{\infty}}\left\|\Delta \psi_{q}\right\|_{L^{\infty}} .
$$

The derivative of the flow equation with respecyt to $x$ and the use of Gronwall and Bernstein inequalities gives

$$
\begin{aligned}
\left\|\nabla^{2} \psi_{q}(t)\right\|_{L^{\infty}} & \lesssim e^{C V(t)} \int_{0}^{t}\left\|\nabla^{2} S_{q-1} v(\tau)\right\|_{L^{\infty}} d \tau \\
& \lesssim e^{C V(t)} 2^{q}
\end{aligned}
$$


Combining both last estimates we obtain

$$
\left\|\Delta g_{q}\right\|_{L^{\infty}} \lesssim e^{C V(t)} 2^{2 q}\left\|f_{q}\right\|_{L^{\infty}} .
$$

Putting together (5) and (7) we conclude that

$$
\left\|J_{q}^{1}(t)\right\|_{L^{\infty}} \lesssim e^{C V(t)}\left(e^{C V(t)}-1\right) 2^{q}\left\|f_{q}\right\|_{L^{\infty}} .
$$

Let us now turn to the second term $J_{q}^{2}$. The integral term can be estimated from (2) as follows

$$
\int_{\mathbb{R}^{2}}|h|^{-\frac{5}{2}}\left\|g_{q}(\cdot)-g_{q}(\cdot-h)\right\|_{L^{\infty}} d h \lesssim\left\|g_{q}\right\|_{\dot{B}_{\infty, 1}^{\frac{1}{2}}}
$$

According to classical composition result we write

$$
\begin{aligned}
\left\|g_{q}(t)\right\|_{\dot{B}_{\infty, 1}^{\frac{1}{2}}} & \lesssim\left\|\nabla \psi_{q}\right\|_{L^{\infty}}^{\frac{1}{2}}\left\|f_{q}\right\|_{\dot{B}_{\infty, 1}^{\frac{1}{2}}} \\
& \lesssim e^{C V(t)} 2^{q \frac{1}{2}}\left\|f_{q}\right\|_{L^{\infty}}
\end{aligned}
$$

In order to estimate $\bar{\psi}_{q}$ we use the interpolation inequality

$$
\left\|\bar{\psi}_{q}(\cdot, h)\right\|_{\dot{B}_{\infty, 1}^{\frac{1}{2}}} \lesssim\left\|\bar{\psi}_{q}(\cdot, h)\right\|_{L^{\infty}}^{\frac{1}{2}}\left\|\nabla_{x} \bar{\psi}_{q}(\cdot, h)\right\|_{L^{\infty}}^{\frac{1}{2}} .
$$

This leads in view of (5) to

$$
\left\|\bar{\psi}_{q}(\cdot, h)\right\|_{\dot{B}_{\infty, 1}^{\frac{1}{2}}} \leq C e^{C V(t)}\left(e^{C V(t)}-1\right)^{\frac{1}{2}}\left\|\nabla_{x} \bar{\psi}_{q}(\cdot, h)\right\|_{L^{\infty}}^{\frac{1}{2}} .
$$

The derivative of $\bar{\psi}_{q}$ with respect to $x$ yields

$$
\begin{aligned}
\left|\nabla_{x} \bar{\psi}_{q}(x, h)\right| & \lesssim \frac{|h|^{\frac{7}{2}}}{\left|\psi_{q}(x)-\psi_{q}(x-h)\right|^{\frac{7}{2}}} \frac{\left|\nabla_{x} \psi_{q}(x)-\nabla_{x} \psi_{q}(x-h)\right|}{|h|} \\
& \lesssim\left\|\nabla \psi_{q}^{-1}\right\|_{L^{\frac{7}{2}}}\left\|\nabla^{2} \psi_{q}\right\|_{L^{\infty}} .
\end{aligned}
$$

Combining (44) and (6) we obtain

$$
\left\|\nabla_{x} \bar{\psi}_{q}(t)\right\|_{L^{\infty}\left(\mathbb{R}^{4}\right)} \lesssim e^{C V(t)} 2^{q} .
$$

Plugging (10) into (9) we find

$$
\left\|\bar{\psi}_{q}(\cdot, h)\right\|_{\dot{B}_{\infty, 1}^{\frac{1}{2}}} \lesssim e^{C V(t)} V^{\frac{1}{2}}(t) 2^{\frac{q}{2}} .
$$

Thus we deduce from (11) and (8) that

$$
\left\|J_{q}^{2}(t)\right\|_{L^{\infty}} \leq C e^{C V(t)} V^{\frac{1}{2}}(t) 2^{q}\left\|f_{q}(t)\right\|_{L^{\infty}} .
$$

This achieves the proof. 


\section{Proof of Theorem 1.2}

The Fourier localized function $\theta_{q}:=\Delta_{q} \theta$ satisfies

(12) $\partial_{t} \theta_{q}+S_{q-1} v \cdot \nabla \theta_{q}+|\mathrm{D}| \theta_{q}=-\left[\Delta_{q}, v \cdot \nabla\right] \theta+\left(S_{q-1} v-v\right) \cdot \nabla \theta_{q}+f_{q}:=\mathcal{R}_{q}$.

Let $\psi_{q}$ denote the flow of the velocity $S_{q-1} v$ and set

$$
\bar{\theta}_{q}(t, x)=\theta_{q}\left(t, \psi_{q}(t, x)\right) \quad \text { and } \quad \overline{\mathcal{R}}_{q}(t, x)=\mathcal{R}_{q}\left(t, \psi_{q}(t, x)\right) .
$$

Since $\psi_{q}$ is an homeomophism, then

$$
\left\|\overline{\mathcal{R}}_{q}\right\|_{L^{\infty}} \leq\left\|\left[\Delta_{q}, v \cdot \nabla\right] \theta\right\|_{L^{\infty}}+\left\|\left(S_{q-1} v-v\right) \cdot \nabla \theta_{q}\right\|_{L^{\infty}}+\left\|f_{q}\right\|_{L^{\infty}} .
$$

It is not hard to check that the function $\bar{\theta}_{q}$ satisfies

$$
\partial_{t} \bar{\theta}_{q}+|\mathrm{D}| \bar{\theta}_{q}=|\mathrm{D}|\left(\theta_{q} \circ \psi_{q}\right)-\left(|\mathrm{D}| \theta_{q}\right) \circ \psi_{q}+\overline{\mathcal{R}}_{q}:=\overline{\mathcal{R}}_{q}^{1} .
$$

From Proposition 3.1 we find that for $q \in \mathbb{Z}$

$$
\left\||\mathrm{D}|\left(\theta_{q} \circ \psi_{q}\right)-\left(|\mathrm{D}| \theta_{q}\right) \circ \psi_{q}\right\|_{L^{\infty}} \lesssim e^{C V(t)} V^{\frac{1}{2}}(t) 2^{q}\left\|\theta_{q}(t)\right\|_{L^{\infty}} .
$$

where $V(t):=\|\nabla v\|_{L_{t}^{1} L^{\infty}}$. Putting together (13) and (15) yields

$$
\begin{aligned}
\left\|\overline{\mathcal{R}}_{q}^{1}(t)\right\|_{L^{\infty}} \lesssim\left\|f_{q}(t)\right\|_{L^{\infty}} & +\left\|\left(S_{q-1} v-v\right) \cdot \nabla \theta_{q}\right\|_{L^{\infty}}+\left\|\left[\Delta_{q}, v \cdot \nabla\right] \theta\right\|_{L^{\infty}} \\
& +e^{C V(t)} V^{\frac{1}{2}}(t) 2^{q}\left\|\theta_{q}(t)\right\|_{L^{\infty}} .
\end{aligned}
$$

Applying the operator $\Delta_{j}$ to the equation (14) and using Proposition 2.6

$$
\begin{aligned}
\left\|\Delta_{j} \bar{\theta}_{q}(t)\right\|_{L^{\infty}} & \lesssim e^{-c t 2^{j}}\left\|\Delta_{j} \theta_{q}^{0}\right\|_{L^{\infty}}+\int_{0}^{t} e^{-c(t-\tau) 2^{j}}\left\|f_{q}(\tau)\right\|_{L^{\infty}} d \tau \\
& +e^{C V(t)} V^{\frac{1}{2}}(t) 2^{q} \int_{0}^{t} e^{-c(t-\tau) 2^{j}}\left\|\theta_{q}(\tau)\right\|_{L^{\infty}} d \tau \\
& +\int_{0}^{t} e^{-c(t-\tau) 2^{j}}\left\|\left[\Delta_{q}, v \cdot \nabla\right] \theta(\tau)\right\|_{L^{\infty}} d \tau \\
& +\int_{0}^{t} e^{-c(t-\tau) 2^{j}}\left\|\left(S_{q-1} v-v\right) \cdot \nabla \theta_{q}(\tau)\right\|_{L^{\infty}} d \tau
\end{aligned}
$$

Integrating this estimate with respect to the time and using Young inequality

$$
\begin{aligned}
\left\|\Delta_{j} \bar{\theta}_{q}\right\|_{L_{t}^{r} L^{\infty}} & \lesssim 2^{-\frac{j}{r}}\left(1-e^{-c r t 2^{j}}\right)^{\frac{1}{r}}\left\|\Delta_{j} \theta_{q}^{0}\right\|_{L^{\infty}}+2^{-j\left(1+\frac{1}{r}-\frac{1}{r}\right)}\left\|f_{q}\right\|_{L_{t}^{r} L^{\infty}} \\
& +e^{C V(t)} V^{\frac{1}{2}}(t) 2^{(q-j)}\left\|\theta_{q}\right\|_{L_{t}^{r} L^{\infty}} \\
& +2^{-\frac{j}{r}} \int_{0}^{t}\left\|\left[\Delta_{q}, v \cdot \nabla\right] \theta(\tau)\right\|_{L^{\infty}} d \tau \\
& +2^{-\frac{j}{r}} \int_{0}^{t}\left\|\left(S_{q-1} v-v\right) \cdot \nabla \theta_{q}(\tau)\right\|_{L^{\infty}} d \tau .
\end{aligned}
$$


Since the flow $\psi$ is an homeomorphism then one writes

$$
\begin{aligned}
& 2^{q\left(s+\frac{1}{r}\right)}\left\|\theta_{q}\right\|_{L_{t}^{r} L^{\infty}}=2^{q\left(s+\frac{1}{r}\right)}\left\|\bar{\theta}_{q}\right\|_{L_{t}^{r} L^{\infty}} \\
\leq & 2^{q\left(s+\frac{1}{r}\right)}\left(\sum_{|j-q|>N}\left\|\Delta_{j} \bar{\theta}_{q}\right\|_{L_{t}^{r} L^{\infty}}+\sum_{|j-q| \leq N}\left\|\Delta_{j} \bar{\theta}_{q}\right\|_{L_{t}^{r} L^{\infty}}\right) \\
:= & \mathrm{I}+\mathrm{II} .
\end{aligned}
$$

To estimate the term I we make appeal to Lemma 2.4

$$
\begin{aligned}
\left\|\Delta_{j} \bar{\theta}_{q}\right\|_{L_{t}^{r} L^{\infty}} & \lesssim 2^{-|q-j|} e^{C \int_{0}^{t}\|\nabla v(\tau)\|_{L^{\infty}} d \tau}\left\|\theta_{q}\right\|_{L_{t}^{r} L^{\infty}} \\
& \leq C 2^{-|q-j|} e^{C V(t)}\left\|\theta_{q}\right\|_{L_{t}^{r} L^{\infty}} .
\end{aligned}
$$

Therefore we get

$$
\mathrm{I} \leq C 2^{-N} e^{C V(t)} 2^{q\left(s+\frac{1}{r}\right)}\left\|\theta_{q}\right\|_{L_{t}^{r} L^{\infty}} .
$$

In order to bound the second term II we use (18)

$$
\begin{aligned}
\mathrm{II} & \lesssim\left(1-e^{-c r t 2^{q}}\right)^{\frac{1}{r}} 2^{q s}\left\|\theta_{q}^{0}\right\|_{L^{\infty}}+2^{N\left(\frac{1}{r}+1-\frac{1}{r}\right)} 2^{q\left(s+\frac{1}{r}-1\right)}\left\|f_{q}\right\|_{L_{t}^{r} L^{\infty}} \\
& +2^{N} e^{C V(t)} V^{\frac{1}{2}}(t) 2^{q\left(s+\frac{1}{r}\right)}\left\|\theta_{q}\right\|_{L_{t}^{r} L^{\infty}} \\
& +2^{\frac{N}{r}} 2^{q s} \int_{0}^{t}\left\|\left[\Delta_{q}, v \cdot \nabla\right] \theta(\tau)\right\|_{L^{\infty}} d \tau \\
& +2^{\frac{N}{r}} 2^{q s} \int_{0}^{t}\left\|\left(S_{q-1} v-v\right) \cdot \nabla \theta_{q}(\tau)\right\|_{L^{\infty}} d \tau .
\end{aligned}
$$

Denote $Z_{q}^{r}(t):=2^{q\left(s+\frac{1}{r}\right)}\left\|\theta_{q}\right\|_{L_{t}^{r} L^{\infty}}$, then we obtain in view of (19) and (20)

$$
\begin{aligned}
Z_{q}^{r}(t) & \leq C\left(1-e^{-c r t 2^{q}}\right)^{\frac{1}{r}} 2^{q s}\left\|\theta_{q}^{0}\right\|_{L^{\infty}}+C 2^{N\left(\frac{1}{r}+1-\frac{1}{r}\right)} 2^{q\left(s+\frac{1}{r}-1\right)}\left\|f_{q}\right\|_{L_{t}^{\bar{r}} L^{\infty}} \\
& +C\left\{2^{N} e^{C V(t)} V^{\frac{1}{2}}(t)+2^{-N} e^{C V(t)}\right\} Z_{q}^{r}(t) \\
& +C 2^{\frac{N}{r}} 2^{q s} \int_{0}^{t}\left\|\left[\Delta_{q}, v \cdot \nabla\right] \theta(\tau)\right\|_{L^{\infty}} d \tau \\
& +C 2^{\frac{N}{r}} 2^{q s} \int_{0}^{t}\left\|\left(S_{q-1} v-v\right) \cdot \nabla \theta_{q}(\tau)\right\|_{L^{\infty}} d \tau .
\end{aligned}
$$

It is easy to check the existence of two absolute constants $N$ and $C_{0}$ such that

$$
V(t) \leq C_{0} \Rightarrow C 2^{-N} e^{C V(t)}+C 2^{N} e^{C V(t)} V^{\frac{1}{2}}(t) \leq \frac{1}{2} .
$$

Thus we obtain under this condition

$$
\begin{aligned}
Z_{q}^{r}(t) & \lesssim\left(1-e^{-c r t 2^{q}}\right)^{\frac{1}{r}} 2^{q s}\left\|\theta_{q}^{0}\right\|_{L^{\infty}}+2^{q\left(s+\frac{1}{\bar{r}}-1\right)}\left\|f_{q}\right\|_{L_{t}^{\bar{r}} L^{\infty}} \\
& +2^{q s} \int_{0}^{t}\left(\left\|\left[\Delta_{q}, v \cdot \nabla\right] \theta(\tau)\right\|_{L^{\infty}}+\left\|\left(S_{q-1} v-v\right) \cdot \nabla \theta_{q}\right\|_{L^{\infty}}\right) d \tau
\end{aligned}
$$


Summing over $q$ and using Lemma 2.3 leads for $V(t) \leq C_{0}$,

$$
\begin{aligned}
\|\theta\|_{\widetilde{L}_{t}^{r} \dot{B}_{\infty, 1}^{s+\frac{1}{r}}} & \lesssim\left\|\theta^{0}\right\|_{\dot{B}_{\infty, 1}^{s}}+\|f\|_{\widetilde{L}_{t}^{\bar{r} \dot{B}_{\infty, 1}^{s+\frac{1}{r}-1}}}+\int_{0}^{t}\|\nabla v(\tau)\|_{L^{\infty}}\|\theta(\tau)\|_{\dot{B}_{\infty, 1}^{s}} d \tau \\
& \lesssim\left\|\theta^{0}\right\|_{\dot{B}_{\infty, 1}^{s}}+\|f\|_{\widetilde{L}_{t}^{\bar{r} \dot{B}_{\infty, 1}^{s+\frac{1}{r}-1}}}+C_{0}\|\theta\|_{L_{t}^{\infty} \dot{B}_{\infty, 1}^{s}} .
\end{aligned}
$$

Let us show how to conclude the proof in the case of $r=\infty$. If $C_{0}$ is sufficiently small then we obtain from (22) the desired estimate:

$$
\|\theta\|_{\widetilde{L}_{t}^{\infty} \dot{B}_{\infty, 1}^{s}} \lesssim\left\|\theta^{0}\right\|_{\dot{B}_{\infty, 1}^{s}}+\|f\|_{\widetilde{L}_{t}^{\bar{r}} \dot{B}_{\infty, 1}^{s+\frac{1}{r}-1}} .
$$

Now for an arbitrary positive time $T$ we take a partition $\left(T_{i}\right)_{i=0}^{M}$ of $[0, T]$, such that $\int_{T_{i}}^{T_{i+1}}\|\nabla v(\tau)\|_{L^{\infty}} d \tau \approx C_{0}$. We can proceed analogously to the above calculus and obtain

$$
\|\theta\|_{\widetilde{L}_{\left[T_{i}, T_{i+1}\right]}^{\infty} \dot{B}_{\infty, 1}^{s}} \lesssim\left\|\theta\left(T_{i}\right)\right\|_{\dot{B}_{\infty, 1}^{s}}+\|f\|_{\widetilde{L}_{\left[T_{i}, T_{i+1}\right]}^{\bar{B}} \dot{B}_{\infty, 1}^{s+\frac{1}{r}-1}} .
$$

An iteration argument leads to

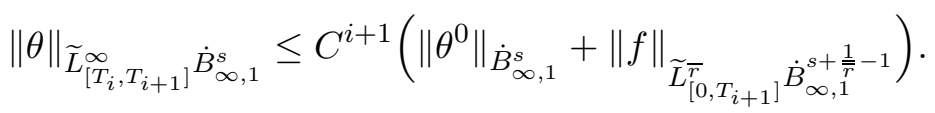

The triangle inequality and the fact that $C_{0} M \simeq 1+V(t)$ give

$$
\|\theta\|_{\widetilde{L}_{T}^{\infty} \dot{B}_{\infty, 1}^{s}} \leq C e^{C \int_{0}^{T}\|\nabla v(\tau)\|_{L^{\infty}}}\left(\left\|\theta^{0}\right\|_{\dot{B}_{\infty, 1}^{s}}+\|f\|_{\widetilde{L}_{T}^{\bar{r} \dot{B}_{\infty, 1}^{s+\frac{1}{r}-1}}}\right) .
$$

Let us now turn to the case of finite $r$. Combining (22) and (23) we obtain under the assumption $V(t) \leq C_{0}$

$$
\|\theta\|_{\widetilde{L}_{t}^{r} \dot{B}_{\infty, 1}^{s+\frac{1}{r}}} \lesssim\left\|\theta^{0}\right\|_{\dot{B}_{\infty, 1}^{s}}+\|f\|_{\widetilde{L}_{t}^{\bar{r}} \dot{B}_{\infty, 1}^{s+\frac{1}{r}-1}}
$$

This gives the result for a short time and as for the case $r=\infty$ we obtain the required global estimate.

Concerning the last estimate of Theorem 1.2, we use in the commutator term of (21) the last part of Lemma 2.3 .

\section{Proof of Theorem 1.1}

The proof is divided into two parts: in the first one we construct local unique solution and we give a criteria of global existence. However we discuss in the second part the global existence by reproducing the same idea of [16].

5.1. Local existence. We aim to prove the following result.

Proposition 5.1. Given any $\theta^{0} \in \dot{B}_{\infty, 1}^{0}$, there is $T>0$ such that the $(\mathrm{QG})_{\frac{1}{2}}$ equation has a unique solution $\theta$ with

$$
\theta \in \widetilde{L}_{T}^{\infty} \dot{B}_{\infty, 1}^{0} \cap L_{T}^{1} \dot{B}_{\infty, 1}^{1} .
$$

Moreover for all $\beta \in \mathbb{R}_{+}$, we have $t^{\beta} \theta \in \widetilde{L}_{T}^{\infty} \dot{B}_{\infty, 1}^{\beta}$. 
Proof. The existence is based on Theorem 1.2 and an iterative method. We denote $\theta_{0}(t, x):=e^{-t|\mathrm{D}|} \theta^{0}(x), v_{0}:=\left(-R_{2} \theta_{0}, R_{1} \theta_{0}\right)$ and $\theta_{n+1}$ the solution of the linear system

$$
\left\{\begin{array}{l}
\partial_{t} \theta_{n+1}+v_{n} \cdot \nabla \theta_{n+1}+|\mathrm{D}| \theta_{n+1}=0 \\
v_{n}=\left(-R_{2} \theta_{n}, R_{1} \theta_{n}\right) \\
\theta_{n+1 \mid t=0}=\theta^{0}
\end{array}\right.
$$

Since $\theta_{0} \in L^{1}\left(\mathbb{R}_{+} ; \dot{B}_{\infty, 1}^{1}\right)$ and from the continuity of Riesz transforms in the homogeneous Besov spaces we find $v_{0} \in L^{1}\left(\mathbb{R}_{+} ; \dot{B}_{\infty, 1}^{1}\right)$. Thus by iteration and thanks to Theorem [1.2, one deduces that $\forall n \in \mathbb{N}$,

$$
\theta_{n} \in \widetilde{L}^{\infty}\left(\mathbb{R}_{+} ; \dot{B}_{\infty, 1}^{0}\right) \cap L^{1}\left(\mathbb{R}_{+} ; \dot{B}_{\infty, 1}^{1}\right) .
$$

Step 1: uniform bounds.

Now we intend to obtain uniform bounds, with respect to the parameter $n$, for some $T>0$ independent of $n$.

By (21), we have for all $T \geq 0$ such that

$$
\int_{0}^{T}\left\|\theta_{n}(\tau)\right\|_{\dot{B}_{\infty, 1}^{1}} d \tau \leq C_{1}\left(:=C C_{0}\right)
$$

the following estimate

$$
\begin{aligned}
\left\|\theta_{n+1}\right\|_{\widetilde{L}_{T}^{2} \dot{B}_{\infty, 1}^{\frac{1}{2}}} & +\left\|\theta_{n+1}\right\|_{L_{T}^{1} \dot{B}_{\infty, 1}^{1}} \lesssim \sum_{q \in \mathbb{Z}}\left(1-e^{-c T 2^{q}}\right)^{\frac{1}{2}}\left\|\Delta_{q} \theta^{0}\right\|_{L^{\infty}} \\
& +\sum_{q \in \mathbb{Z}} \int_{0}^{T}\left\|\left[\Delta_{q}, v_{n} \cdot \nabla\right] \theta_{n+1}(\tau)\right\|_{L^{\infty}} d \tau \\
& +\sum_{q \in \mathbb{Z}} \int_{0}^{T}\left\|\left(S_{q-1} v_{n}-v_{n}\right) \cdot \nabla \Delta_{q} \theta_{n+1}(\tau)\right\|_{L^{\infty}} d \tau .
\end{aligned}
$$

Since $\operatorname{div} v_{n}=0$, then Lemma 2.3 combined with the continuity of Riesz transforms gives

$$
\begin{aligned}
\sum_{q \in \mathbb{Z}} \int_{0}^{T}\left\|\left[\Delta_{q}, v_{n} \cdot \nabla\right] \theta_{n+1}(\tau)\right\|_{L^{\infty}} d \tau & \lesssim\left\|v_{n}\right\|_{\widetilde{L}_{T}^{2} \dot{B}_{\infty, \infty}^{\frac{1}{2}}}\left\|\theta_{n+1}\right\|_{\widetilde{L}_{T}^{2} \dot{B}_{\infty, 1}^{\frac{1}{2}}} \\
& \lesssim\left\|\theta_{n}\right\|_{\widetilde{L}_{T}^{2} \dot{B}_{\infty, \infty}^{\frac{1}{2}}}\left\|\theta_{n+1}\right\|_{\widetilde{L}_{T}^{2} \dot{B}_{\infty, 1}^{\frac{1}{2}}} .
\end{aligned}
$$

We deduce from Hölder and Young inequalities

$$
\begin{gathered}
\sum_{q \in \mathbb{Z}} \int_{0}^{t}\left\|\left(S_{q} v_{n}-v_{n}\right) \cdot \nabla \Delta_{q} \theta_{n+1}\right\|_{L^{\infty}} d \tau \lesssim \sum_{q \in \mathbb{Z}} 2^{q}\left\|\Delta_{q} \theta_{n+1}\right\|_{L_{t}^{2} L^{\infty}}\left\|S_{q} v_{n}-v_{n}\right\|_{L_{t}^{2} L^{\infty}} \\
\lesssim \sum_{q \in \mathbb{Z}} 2^{\frac{1}{2} q}\left\|\Delta_{q} \theta_{n+1}\right\|_{L_{t}^{2} L^{\infty}} \sum_{k \geq q} 2^{\frac{1}{2}(q-k)} 2^{\frac{1}{2} k}\left\|\Delta_{k} v_{n}\right\|_{L_{t}^{2} L^{\infty}} \\
\lesssim\left\|\theta_{n+1}\right\|_{\widetilde{L}_{t}^{2} \dot{B}_{\infty, 1}^{\frac{1}{2}}}\left\|\theta_{n}\right\|_{\widetilde{L}_{t}^{2} \dot{B}_{\infty, \infty}^{\frac{1}{2}}} \cdot
\end{gathered}
$$


Therefore we obtain from the above inequalities

$$
\begin{aligned}
& \left\|\theta_{n+1}\right\|_{\widetilde{L}_{t}^{2} \dot{B}_{\infty, 1}^{\frac{1}{2}}}+\left\|\theta_{n+1}\right\|_{L_{t}^{1} \dot{B}_{\infty, 1}^{1}} \lesssim \sum_{q \in \mathbb{Z}}\left(1-e^{-c t 2^{q}}\right)^{\frac{1}{2}}\left\|\Delta_{q} \theta^{0}\right\|_{L^{\infty}} \\
& +\left\|\theta_{n+1}\right\|_{\widetilde{L}_{t}^{2} \dot{B}_{\infty, 1}^{\frac{1}{2}}}\left\|\theta_{n}\right\|_{\widetilde{L}_{t}^{2} \dot{B}_{\infty, 1}^{\frac{1}{2}}} .
\end{aligned}
$$

Thus there exists an absolute constant $\varepsilon_{0}>0$ such that, if

$$
\sum_{q \in \mathbb{Z}}\left(1-e^{-c T 2^{q}}\right)^{\frac{1}{2}}\left\|\Delta_{q} \theta^{0}\right\|_{L^{\infty}} \leq \varepsilon_{0}
$$

then

$$
\left\|\theta_{n+1}\right\|_{\widetilde{L}_{T}^{2} \dot{B}_{\infty, 1}^{\frac{1}{2}}}+\left\|\theta_{n+1}\right\|_{L_{T}^{1} \dot{B}_{\infty, 1}^{1}} \leq 2 \varepsilon_{0} .
$$

The existence of $T>0$ is due to Lebesgue theorem.

Hence, by using the estimate

$$
\int_{0}^{T}\|\nabla v(\tau)\|_{L^{\infty}} d \tau \lesssim \int_{0}^{T}\|\theta(\tau)\|_{\dot{B}_{\infty, 1}^{1}} d \tau
$$

and Theorem 1.2 we obtain

$$
\left\|\theta_{n+1}\right\|_{\widetilde{L}_{T}^{\infty} \dot{B}_{\infty, 1}^{0}} \lesssim\left\|\theta^{0}\right\|_{\dot{B}_{\infty, 1}^{0}} .
$$

Thus we prove that the sequence $\left(v_{n}, \theta_{n}\right)_{n \in \mathbb{N}}$ is uniformly bounded in the space $\widetilde{L}_{T}^{\infty} \dot{B}_{\infty, 1}^{0} \cap L_{T}^{1} \dot{B}_{\infty, 1}^{1}$.

Step 2: strong convergence.

We will prove that the sequence $\left(v_{n}, \theta_{n}\right)$ is of Cauchy in $\widetilde{L}_{T}^{\infty} \dot{B}_{\infty, 1}^{0}$.

Let $(n, m) \in \mathbb{N}^{2}, \theta_{n, m}=: \theta_{n+1}-\theta_{m+1}$ and $v_{n, m}:=v_{n}-v_{m}$, then

$$
\left\{\begin{array}{l}
\partial_{t} \theta_{n, m}+v_{n} \cdot \nabla \theta_{n, m}+|\mathrm{D}| \theta_{n, m}=-v_{n, m} \cdot \nabla \theta_{m+1} \\
\theta_{n, m \mid t=0}=0 .
\end{array}\right.
$$

Applying Theorem 1.2 to this equation gives

$$
\left\|\theta_{n, m}\right\|_{\widetilde{L}_{t}^{\infty} \dot{B}_{\infty, 1}^{0}} \leq C e^{C\left\|\theta_{n}\right\|_{L_{t}^{1} \dot{B}_{\infty, 1}^{1}}} \int_{0}^{t}\left\|v_{n, m} \cdot \nabla \theta_{m+1}(\tau)\right\|_{\dot{B}_{\infty, 1}^{0}} d \tau
$$

Thanks to Bony's decomposition [1], the embedding $\dot{B}_{\infty, 1}^{0} \hookrightarrow L^{\infty}$ and the fact that $\operatorname{div} v_{n, m}=0$

$$
\left\|v_{n, m} \cdot \nabla \theta_{m+1}\right\|_{\dot{B}_{\infty, 1}^{0}} \lesssim\left\|v_{n, m}\right\|_{\dot{B}_{\infty, 1}^{0}}\left\|\theta_{m+1}\right\|_{\dot{B}_{\infty, 1}^{1}} .
$$

Since Riesz transforms map continuously $\dot{B}_{\infty, 1}^{0}$ into itself, then we get

$$
\left\|v_{n, m} \cdot \nabla \theta_{m+1}\right\|_{\dot{B}_{\infty, 1}^{0}} \lesssim\left\|\theta_{n-1, m-1}\right\|_{\dot{B}_{\infty, 1}^{0}}\left\|\theta_{m+1}\right\|_{\dot{B}_{\infty, 1}^{1}} .
$$

Thus we infer

$$
\left\|\theta_{n, m}\right\|_{\widetilde{L}_{t}^{\infty} \dot{B}_{\infty, 1}^{0}} \leq C\left\|\theta_{n-1, m-1}\right\|_{\widetilde{L}_{t}^{\infty} \dot{B}_{\infty, 1}^{0}} e^{C\left\|\theta_{n}\right\|_{L_{t}^{1} \dot{B}_{\infty, 1}^{1}}} \int_{0}^{t}\left\|\theta_{m+1}(\tau)\right\|_{\dot{B}_{\infty, 1}^{1}} d \tau .
$$


According to the inequality (28) one can choose $\varepsilon_{0}$ small such that

$$
\left\|\theta_{n, m}\right\|_{\widetilde{L}_{T}^{\infty} \dot{B}_{\infty, 1}^{0}} \leq \eta\left\|\theta_{n-1, m-1}\right\|_{\widetilde{L}_{T}^{\infty} \dot{B}_{\infty, 1}^{0}}
$$

with $\eta<1$. Let us suppose that $n \geq m$, then by induction one finds

$$
\left\|\theta_{n, m}\right\|_{\widetilde{L}_{T}^{\infty} \dot{B}_{\infty, 1}^{0}} \lesssim \eta^{m}\left\|\theta^{0}\right\|_{\dot{B}_{\infty, 1}^{0}}
$$

Thus $\left(\theta_{n}\right)_{n \in \mathbb{N}}$ is a Cauchy sequence in $\widetilde{L}_{T}^{\infty} \dot{B}_{\infty, 1}^{0}$. Then there exists $\theta \in \widetilde{L}_{t}^{\infty} \dot{B}_{\infty, 1}^{0}$ such that $\theta_{n}$ converges strongly to $\theta$ in $\widetilde{L}_{t}^{\infty} \dot{B}_{\infty, 1}^{0}$. Moreover Fatou lemma and inequality (28) imply that $\theta \in L_{t}^{1} \dot{B}_{\infty, 1}^{1}$. These informations allow us to pass to the limit into the equation.

\section{Step 3: Uniqueness.}

Let us denote $X_{T}:=L_{T}^{\infty} \dot{B}_{\infty, 1}^{0} \cap L_{T}^{1} \dot{B}_{\infty, 1}^{1}$ and $\theta_{i}, i=1,2$ ( $v_{i}$ the corresponding velocity) be two solutions of the $(\mathrm{QG})_{\frac{1}{2}}$ equation with the same initial data and belonging to the space $X_{T}$. We set $\theta_{1,2}=\theta_{1}-\theta_{2}$ and $v_{1,2}=v_{1}-v_{2}$, then it is plain that

$$
\partial_{t} \theta_{1,2}+v^{1} \cdot \nabla \theta_{1,2}+|\mathrm{D}| \theta_{1,2}=-v_{1,2} \cdot \nabla \theta^{2}, \theta_{1,2} \mid t=0=0 .
$$

Thanks to the inequalities (29) and (31), we have

$$
\left\|\theta_{1,2}\right\|_{\widetilde{L}_{t}^{\infty} \dot{B}_{\infty, 1}^{0}} \leq C e^{C\left\|\theta_{1}\right\|_{L_{t}^{1} \dot{B}_{\infty, 1}^{1}}} \int_{0}^{t}\left\|\theta_{1,2}\right\|_{\widetilde{L}_{\tau}^{\infty} \dot{B}_{\infty, 1}^{0}}\left\|\theta_{2}(\tau)\right\|_{\dot{B}_{\infty, 1}^{1}} d \tau
$$

Thus Gronwall's inequality gives the desired result.

Step 4: smoothing effect.

We will show the precise estimate: for all $\beta \in \mathbb{R}_{+}$we have

$$
\left\|t^{\beta} \theta(t)\right\|_{\widetilde{L}_{T}^{\infty} \dot{B}_{\infty, 1}^{\beta}} \leq C_{\beta} e^{C(\beta+1)\|\theta\|_{L_{T}^{1} \dot{B}_{\infty, 1}^{1}}\|\theta\|_{\widetilde{L}_{T}^{\infty} \dot{B}_{\infty, 1}^{0}} .}
$$

It is clear that

$$
\left\{\begin{array}{l}
\partial_{t}\left(t^{\beta} \theta\right)+v \cdot \nabla\left(t^{\beta} \theta\right)+|\mathrm{D}|\left(t^{\beta} \theta\right)=\beta t^{\beta-1} \theta \\
\left(t^{\beta} \theta\right)_{\mid t=0}=0 .
\end{array}\right.
$$

We will proceed by induction and start the proof with the case $\beta \in \mathbb{N}$.

For $\beta=1$, we apply Theorem 1.2 with $\bar{r}=+\infty$,

$$
\|t \theta(t)\|_{\widetilde{L}_{T}^{\infty} \dot{B}_{\infty, 1}^{1}} \lesssim e^{C\|\theta\|_{L_{T}^{1} \dot{B}_{\infty, 1}^{1}}\|\theta\|_{\widetilde{L}_{T}^{\infty} \dot{B}_{\infty, 1}^{0}} .}
$$

Assume (32) holds for degree $n$; we will prove it for $n+1$.

Applying Theorem 1.2 to the equation of $t^{n+1} \theta$ we get

$$
\begin{aligned}
\left\|t^{n+1} \theta(t)\right\|_{\widetilde{L}_{T}^{\infty} \dot{B}_{\infty, 1}^{n+1}} & \leq C(n+1) e^{C\|\theta\|_{L_{T}^{1} \dot{B}_{\infty, 1}^{1}}}\left\|t^{n} \theta\right\|_{\widetilde{L}_{T}^{\infty} \dot{B}_{\infty, 1}^{n}} \\
& \leq C_{n} e^{C(n+2)\|\theta\|_{L_{T}^{1} \dot{B}_{\infty, 1}^{1}}\|\theta\|_{\widetilde{L}_{T}^{\infty} \dot{B}_{\infty, 1}^{0}}} .
\end{aligned}
$$


For $\beta \in \mathbb{R}_{+}$, we have $[\beta] \leq \beta<[\beta]+1$ and by interpolation, one has

$$
\left\|t^{\beta} \theta\right\|_{\widetilde{L}_{T}^{\infty}\left(\dot{B}_{\infty, 1}^{\beta}\right)} \lesssim\left\|t^{[\beta]} \theta\right\|_{\widetilde{L}_{T}^{\infty}\left(\dot{B}_{\infty, 1}^{[\beta]}\right)}^{1+[\beta]-\beta}\left\|t^{[\beta]+1} \theta\right\|_{\widetilde{L}_{T}^{\infty}\left(\dot{B}_{\infty, 1}^{[\beta]+1}\right)}^{\beta-[\beta]} .
$$

This completes the proof.

5.2. Blowup Criteria. The main result of this paragraph is:

Proposition 5.2. Let $T^{*}$ be the maximum local existence time of $\theta$ in $\widetilde{L}_{T}^{\infty} \dot{B}_{\infty, 1}^{0} \cap L_{T}^{1} \dot{B}_{\infty, 1}^{1}$. There exists an absolute constant $\varepsilon_{0}>0$ such that if $T^{*}<\infty$, then

$$
\liminf _{t \rightarrow T^{*}}\left(T^{*}-t\right)\|\nabla \theta(t)\|_{L^{\infty}} \geq \varepsilon_{0} .
$$

Proof. From local existence theory and especially (27) we see that if $T^{*}<\infty$, then necessary

$$
\liminf _{t \rightarrow T^{*}} \sum_{q \in \mathbb{Z}}\left(1-e^{-c\left(T^{*}-t\right) 2^{q}}\right)^{\frac{1}{2}}\left\|\theta_{q}(t)\right\|_{L^{\infty}} \geq \varepsilon_{0},
$$

otherwise we can continue the solution over $T^{*}$. It follows that

$$
\liminf _{t \rightarrow T^{*}} \sum_{q \in \mathbb{Z}}\left(1-e^{-c\left(T^{*}-t\right) 2^{q}}\right)^{\frac{1}{2}} \sup _{t \leq T^{*}}\left\|\theta_{q}(t)\right\|_{L^{\infty}} \geq \varepsilon_{0}
$$

Consequently we obtain from Lebesgue theorem

$$
\|\theta\|_{\widetilde{L}_{T^{*}}^{\infty}\left(\dot{B}_{\infty, 1}^{0}\right)}=\infty .
$$

Using Bernstein inequality and the fact that $\left\|\theta_{q}\right\|_{L^{\infty}} \lesssim\left\|\theta^{0}\right\|_{L^{\infty}}$, we have

$$
\begin{aligned}
\varepsilon_{0} \leq \liminf _{t \rightarrow T^{*}} & \left\{\sum_{q \leq N}\left(1-e^{-c\left(T^{*}-t\right) 2^{q}}\right)^{\frac{1}{2}}\left\|\theta_{q}(t)\right\|_{L^{\infty}}\right. \\
& \left.+\sum_{q \geq N}\left(1-e^{-c\left(T^{*}-t\right) 2^{q}}\right)^{\frac{1}{2}}\left\|\theta_{q}(t)\right\|_{L^{\infty}}\right\} \\
& \lesssim \liminf _{t \rightarrow T^{*}}\left\{\left(T^{*}-t\right)^{\frac{1}{2}}\left\|\theta^{0}\right\|_{L^{\infty}} \sum_{q \leq N} 2^{q / 2}+\|\nabla \theta(t)\|_{L^{\infty}} \sum_{q \geq N} 2^{-q}\right\} \\
& \lesssim \liminf _{t \rightarrow T^{*}}\left\{\left(T^{*}-t\right)\left\|\theta^{0}\right\|_{L^{\infty}} 2^{N}+\|\nabla \theta(t)\|_{L^{\infty}} 2^{-N}\right\} .
\end{aligned}
$$

Choosing judiciously $N$ we obtain the desired result.

5.3. Global existence. We will use the idea of [16]. Let $T^{*}$ be the maximal time existence of the solution in the space $\widetilde{L}_{\text {loc }}^{\infty}\left(\left[0, T^{*}\left[, \dot{B}_{\infty, 1}^{0}\right) \cap\right.\right.$ $L_{\mathrm{loc}}^{1}\left(\left[0, T^{*}\left[, \dot{B}_{\infty, 1}^{1}\right)\right.\right.$. From the local existence, there exists $T_{0}>0$ such that

$$
\forall t \in\left[0, T_{0}\right], t\|\nabla \theta(t)\|_{L^{\infty}} \leq C\left\|\theta^{0}\right\|_{\dot{B}_{\infty, 1}^{0}} .
$$

Let $\lambda$ be a real positive number that will be fixed later and $\left.T_{1} \in\right] 0, T_{0}[$. We define the set

$I:=\left\{T \in\left[T_{1}, T^{*}\left[; \forall t \in\left[T_{1}, T\right], \forall x \neq y \in \mathbb{R}^{2},|\theta(t, x)-\theta(t, y)|<\omega_{\lambda}(|x-y|)\right\}\right.\right.$, 
where

$$
\omega: \mathbb{R}_{+} \longrightarrow \mathbb{R}_{+},
$$

is strictly increasing, concave, $\omega(0)=0, \omega^{\prime}(0)<+\infty, \lim _{\xi \longrightarrow 0^{+}} \omega^{\prime \prime}(\xi)=-\infty$ and

$$
\omega_{\lambda}(|x-y|)=\omega(\lambda|x-y|) .
$$

The function $\omega$ is a modulus of continuity chosen as in [16]. We shall first check that $I$ is nonempty. It suffices for this purpose to prove that $T_{1}$ belongs to $I$ under suitable conditions over $\lambda$. Let $C_{0}$ be a large positive number such that

$$
\omega\left(C_{0}\right)>2\left\|\theta^{0}\right\|_{L^{\infty}} .
$$

Since $\omega$ is strictly non-decreasing function then we get from maximum principle

$$
\forall x, y ; \lambda|x-y| \geq C_{0} \Rightarrow\left|\theta\left(T_{1}, x\right)-\theta\left(T_{1}, y\right)\right| \leq 2\left\|\theta^{0}\right\|_{L^{\infty}}<\omega_{\lambda}(|x-y|) .
$$

On the other hand we have from Mean Value Theorem

$$
\left|\theta\left(T_{1}, x\right)-\theta\left(T_{1}, y\right)\right| \leq|x-y|\left\|\nabla \theta\left(T_{1}\right)\right\|_{L^{\infty}} .
$$

Let $0<\delta_{0}<C_{0}$. Then using the concavity of $\omega$ one obtains

$$
\lambda|x-y| \leq \delta_{0} \Rightarrow \omega_{\lambda}(|x-y|) \geq \frac{\omega\left(\delta_{0}\right)}{\delta_{0}} \lambda|x-y| .
$$

If we choose $\lambda$ so that

$$
\lambda>\frac{\delta_{0}}{\omega\left(\delta_{0}\right)}\left\|\nabla \theta\left(T_{1}\right)\right\|_{L^{\infty}},
$$

then we get

$$
0<\lambda|x-y| \leq \delta_{0} \Rightarrow\left|\theta\left(T_{1}, x\right)-\theta\left(T_{1}, y\right)\right|<\omega_{\lambda}(|x-y|) .
$$

Let us now move to the case $\delta_{0} \leq \lambda|x-y| \leq C_{0}$. By an obvious computation we find

$$
\begin{aligned}
\left|\theta\left(T_{1}, x\right)-\theta\left(T_{1}, y\right)\right| & \leq \frac{C_{0}}{\lambda}\left\|\nabla \theta\left(T_{1}\right)\right\|_{L^{\infty}} \quad \text { and } \\
\omega\left(\delta_{0}\right) & \leq \omega(\lambda|x-y|) .
\end{aligned}
$$

Choosing $\lambda$ such that

$$
\lambda>\frac{C_{0}}{\omega\left(\delta_{0}\right)}\left\|\nabla \theta\left(T_{1}\right)\right\|_{L^{\infty}}
$$

Then we obtain

$$
\delta_{0} \leq \lambda|x-y| \leq C_{0} \Rightarrow\left|\theta\left(T_{1}, x\right)-\theta\left(T_{1}, y\right)\right|<\omega_{\lambda}(|x-y|) .
$$

All the preceding conditions over $\lambda$ can be obtained if we take

$$
\lambda=\frac{\omega^{-1}\left(3\left\|\theta^{0}\right\|_{L^{\infty}}\right)}{2\left\|\theta^{0}\right\|_{L^{\infty}}}\left\|\nabla \theta\left(T_{1}\right)\right\|_{L^{\infty}} .
$$

From the construction, the set $I$ is an interval of the form $\left[T_{1}, T_{*}\right)$. We have three possibilities. The first one is $T_{*}=T^{*}$ and in this case we have 
necessary $T^{*}=+\infty$ because the Lipshitz norm of $\theta$ does not blow up. The second one is $T_{*} \in I$ and we will show that is not possible. Indeed, let $C_{0}$ as (33) then for all $t \in\left[T_{1}, T^{*}\right)$

$$
\lambda|x-y| \geq C_{0} \Rightarrow|\theta(t, x)-\theta(t, y)|<\omega_{\lambda}(|x-y|)
$$

Since $\nabla \theta(t)$ belongs to $\left.\left.C(] 0, T^{*}\right) ; \dot{B}_{\infty, 1}^{0}\right)$, then for $\epsilon>0$ there exist $\eta_{0}, R>0$ such that

$$
\begin{gathered}
\forall t \in\left[T_{*}, T_{*}+\eta_{0}\right] \Rightarrow\|\nabla \theta(t)\|_{L^{\infty}} \leq\left\|\nabla \theta\left(T_{*}\right)\right\|_{L^{\infty}}+\epsilon / 2 \quad \text { and } \\
\left\|\nabla \theta\left(T_{*}\right)\right\|_{L^{\infty}\left(B_{(0, R)}^{c}\right)} \leq \epsilon / 2,
\end{gathered}
$$

where $B_{(0, R)}$ is the ball of radius $R$ and with center the origin.

Hence for $\lambda|x-y| \leq C_{0}$ and $x$ or $y \in B_{\left(0, R+\frac{C_{0}}{\lambda}\right)}^{c}$ we have for $t \in\left[T_{*}, T_{*}+\eta_{0}\right]$

$$
\begin{aligned}
|\theta(t, x)-\theta(t, y)| & \leq|x-y|\|\nabla \theta(t)\|_{L^{\infty}\left(B_{(0, R)}^{c}\right)} \\
& \leq \epsilon|x-y| .
\end{aligned}
$$

On the other hand we have from the concavity of $\omega$

$$
\lambda|x-y| \leq C_{0} \Rightarrow \frac{\omega\left(C_{0}\right)}{C_{0}} \lambda|x-y| \leq \omega_{\lambda}(|x-y|)
$$

Thus if we take $\epsilon$ sufficiently small such that

$$
\epsilon<\frac{\omega\left(C_{0}\right)}{C_{0}} \lambda
$$

then we find that

$$
\lambda|x-y| \leq C_{0} ; x \text { or } y \in B_{\left(0, R+\frac{C_{0}}{\lambda}\right)}^{c} \Rightarrow|\theta(t, x)-\theta(t, y)|<\omega_{\lambda}(|x-y|) .
$$

It remains to study the case where $x, y \in B_{\left(0, R+\frac{C_{0}}{\lambda}\right)}$. Since $\left\|\nabla^{2} \theta\left(T_{*}\right)\right\|_{L^{\infty}}$ is finite (see Proposition 5.1) then we get for each $x \in \mathbb{R}^{2}$

$$
\left|\nabla \theta\left(T_{*}, x\right)\right|<\lambda \omega^{\prime}(0) .
$$

For the proof see [25], page 4. From the continuity of $x \longmapsto\left|\nabla \theta\left(T_{*}, x\right)\right|$ we obtain

$$
\left\|\nabla \theta\left(T_{*}\right)\right\|_{L^{\infty}\left(B_{\left(0, R+\frac{C_{0}}{\lambda}\right)}\right)}<\lambda \omega^{\prime}(0)
$$

Let $\delta_{0}<<1$ then using the continuity in time of the quantity $\|\nabla \theta(t)\|_{L^{\infty}}$ one can find $\eta_{1}>0$ such that $\forall t \in\left[T_{*}, T_{*}+\eta_{1}\right]$

$$
\|\nabla \theta(t)\|_{L^{\infty}\left(B, R+\frac{C_{0}}{\lambda}\right)}<\lambda \frac{\omega\left(\delta_{0}\right)}{\delta_{0}} .
$$

For $\lambda|x-y| \leq \delta_{0}$ and $x \neq y$ belonging together to $B_{\left(0, R+\frac{C_{0}}{\lambda}\right)}$ we have

$$
\begin{aligned}
|\theta(t, x)-\theta(t, y)| & \leq|x-y|\|\nabla \theta(t)\|_{L^{\infty}\left(B_{\left(0, R+\frac{C_{0}}{\lambda}\right)}\right)} \\
& <\lambda|x-y| \frac{\omega\left(\delta_{0}\right)}{\delta_{0}} \leq \omega_{\lambda}(|x-y|) .
\end{aligned}
$$


Now for the other case we have

$$
\forall x, y \in B_{\left(0, R+\frac{C_{0}}{\lambda}\right)}, \delta_{0} \leq \lambda|x-y| ;\left|\theta\left(T_{*}, x\right)-\theta\left(T_{*}, y\right)\right|<\omega_{\lambda}(|x-y|),
$$

then we get from a standard compact argument the existence of $\eta_{2}>0$ such that for all $t \in\left[T_{*}, T_{*}+\eta_{2}\right]$

$$
\forall x, y \in B_{\left(0, R+\frac{C_{0}}{\lambda}\right)}, \delta_{0} \leq \lambda|x-y| ;|\theta(t, x)-\theta(t, y)|<\omega_{\lambda}(|x-y|) .
$$

Taking $\eta=\min \left\{\eta_{0}, \eta_{1}, \eta_{2}\right\}$, we obtain that $T_{*}+\eta \in I$ which contradicts the fact that $T_{*}$ is maximal.

The last case that we have to treat is that $T_{*}$ does not belong to $I$. Thus we have by time continuity of $\theta$ the existence of $x \neq y$ such that

$$
\theta\left(T_{*}, x\right)-\theta\left(T_{*}, y\right)=\omega_{\lambda}(\xi), \text { with } \xi=|x-y| .
$$

We will show that this scenario can not occur and more precisely:

$$
f^{\prime}\left(T_{*}\right)<0 \quad \text { where } \quad f(t)=\theta(t, x)-\theta(t, y) .
$$

This is impossible since $f(t) \leq f\left(T_{*}\right), \forall t \in\left[0, T_{*}\right]$. The proof is the same as [16] and for the convenience of the reader we will outline the proof. From the regularity of the solution we see that the $(\mathrm{QG})_{\frac{1}{2}}$ equation can be defined in the classical manner and

$$
f^{\prime}\left(T_{*}\right)=(u \cdot \nabla \theta)\left(T_{*}, x\right)-(u \cdot \nabla \theta)\left(T_{*}, y\right)+|\mathrm{D}| \theta\left(T_{*}, x\right)-|\mathrm{D}| \theta\left(T_{*}, y\right) .
$$

From [16] we have

$$
(u \cdot \nabla \theta)\left(T_{*}, x\right)-(u \cdot \nabla \theta)\left(T_{*}, y\right) \leq \Omega_{\lambda}(\xi) \omega_{\lambda}^{\prime}(\xi),
$$

where

$$
\Omega_{\lambda}(\xi)=C\left(\int_{0}^{\xi} \frac{\omega_{\lambda}(\eta)}{\eta} d \eta+\xi \int_{\xi}^{\infty} \frac{\omega_{\lambda}(\eta)}{\eta^{2}} d \eta\right)=\Omega(\lambda \xi) .
$$

Again from [16]

$$
\begin{aligned}
|\mathrm{D}| \theta\left(T_{*}, x\right)-|\mathrm{D}| \theta\left(T_{*}, y\right) & \leq \frac{1}{\pi} \int_{0}^{\frac{\xi}{2}} \frac{\omega_{\lambda}(\xi+2 \eta)+\omega_{\lambda}(\xi-2 \eta)-2 \omega_{\lambda}(\xi)}{\eta^{2}} d \eta \\
& +\frac{1}{\pi} \int_{\frac{\xi}{2}}^{\infty} \frac{\omega_{\lambda}(2 \eta+\xi)-\omega_{\lambda}(2 \eta-\xi)-2 \omega_{\lambda}(\xi)}{\eta^{2}} d \eta \\
& \leq \lambda \mathcal{I}(\lambda \xi),
\end{aligned}
$$

where

$$
\begin{aligned}
\mathcal{I}(\xi) & =\frac{1}{\pi} \int_{0}^{\frac{\xi}{2}} \frac{\omega(\xi+2 \eta)+\omega(\xi-2 \eta)-2 \omega(\xi)}{\eta^{2}} d \eta \\
& +\frac{1}{\pi} \int_{\frac{\xi}{2}}^{\infty} \frac{\omega(2 \eta+\xi)-\omega(2 \eta-\xi)-2 \omega(\xi)}{\eta^{2}} d \eta
\end{aligned}
$$

Thus we get

$$
f^{\prime}\left(T_{*}\right)=\lambda\left(\Omega \omega^{\prime}+\mathcal{I}\right)(\lambda \xi)
$$


Now, we choose the same function as [16] (see page 5)

$$
\omega(\xi)=\xi-\xi^{\frac{3}{2}}, \text { if } \quad \xi \in[0, \delta]
$$

and

$$
\omega^{\prime}(\xi)=\frac{\gamma}{\xi(4+\log (\xi / \delta))}, \text { if } \quad \xi>\delta,
$$

where $\delta$ and $\gamma$ are small numbers and satisfy $0<\gamma<\delta$. It is shown in [16] that

$$
\Omega(\xi) \omega^{\prime}(\xi)+\mathcal{I}(\xi)<0, \forall \xi \neq 0 .
$$

This yields to $f^{\prime}\left(T_{*}\right)<0$.

Finally we have $T^{*}=+\infty$ and

$$
\forall t \in\left[T_{1},+\infty\right),\|\nabla \theta(t)\|_{L^{\infty}} \leq \lambda .
$$

The value of $\lambda$ is given by (34).

\section{REFERENCES}

[1] J.-M. Bony, Calcul symbolique et propagation des singularités pour les équations aux dérivées partielles non linéaires, Annales de l'école supérieure, 14 (1981), 209-246.

[2] L. Caffarelli and V. Vasseur, Drift diffusion equations with fractional diffusion and the quasi-geostrophic equations, arXiv, math.AP/0608447

[3] D. Chae and J. Lee, Global well-posedness in the supercritical dissipative quasigeostrophic equations, Asymptot. Anal. 38 (2004), no. 3-4, 339-358.

[4] J.-Y. Chemin, Perfect incompressible Fluids, The Clarendon Press, Oxford University Press, New York, 1998.

[5] Q. Chen, C. Miao, Z, Zhang, A new Bernstein's inequality and the $2 D$ dissipative quasi-geostrophic equation, arXiv, math.AP/0607020.

[6] P. Constantin, A. Majda, and E. Tabak, Formation of strong fronts in the $2 D$ quasi-geostrophic thermal active scalar, Nonlinearity, 7 (1994), 1495-1533.

[7] P. Constantin, D. Córdoba and J. Wu, On the critical dissipative quasi-geostrophic equation, Indiana Univ. Math. J., 50 (2001), 97-107.

[8] P. Constantin and J. Wu, Behaviour of solutions of $2 D$ quasi-geostrophic equations, SIAM J. Math. Anal, 30 (1999), 937-948.

[9] A. Córdoba and D. Córdoba, A maximum principle applied to quasi-geostrophic equations, Comm. Math. Phys., 249 (2004), 511-528.

[10] R. Danchin, Density-dependent incompressible viscous fluids in critical spaces, Proc. Roy. Soc. Ed. 133 (2003), 1311-1334.

[11] T. Hmidi, Régularité höldérienne des poches de tourbillon visqueuses, J. Math. Pures Appl. (9) 84 (2005), no. 11, 1455-1495.

[12] T. Hmidi and S. Keraani, Global solutions of the super-critical $2 D$ Q-G equation in Besov spaces, arXiv, math.AP/0611494

[13] N. Ju, Existence and uniqueness of the solution to the dissipative 2D quasigeostrophic equations in the Sobolev space. Comm. Math. Phus. 251 (2004), 365376.

[14] N. Ju, On the two dimensional quasi-geostrophic equations, Indiana Univ. Math. J. 54 (2005), no. 3, 897-926.

[15] N. Ju, Global solutions to the two dimensional quasi-geostrophic equation with critical or super-critical dissipation Math. Ann. 334 (2006), no. 3, 627-642.

[16] A. Kiselev, F. Nazarov and A. Volberg, Global well-posedness for the critical $2 D$ dissipative quasi-geostrophic equation, arXiv, math.AP/0604185 
[17] F. Marchand, P.-G. Lemarié-Rieusset, Solutions auto-similaires non radiales pour l'quation quasi-gostrophique dissipative critique. C. R. Math. Acad. Sci. Paris 341 (2005), no. 9, 535-538.

[18] J. Peetre, New thoughts on Besov spaces, Duke University Mathematical Series 1, Durham N. C. 1976.

[19] J. Pedlosky, Geophysical Fluid Dynamics, Springer-Verlag, New York, 1987.

[20] S. Resnick, Dynamical problem in nonlinear advective partial differential equations, Ph.D. thesis, University of Chicago, 1995

[21] H. Triebel, Theory of function spaces.

[22] M. Vishik, Hydrodynamics in Besov Spaces, Arch. Rational Mech. Anal 145 (1998), 197-214.

[23] J. Wu, Solutions to the 2D quasi-geostrophic equations in Hölder spaces, Nonlinear Analysis, 62 (2005), 579-594.

[24] J. Wu, Global solutions of the $2 D$ dissipative quasi-geostrophic equations in Besov spaces, SIAM J. Math. Anal., 36 (2004/05), no 3, 1014-1030.

[25] Xinwei Yu, Remarks on the global regularity for the super-critical $2 D$ dissipative quasi-geostrophic equation arXiv, math.AP/0611283

IRMAR, Université de Rennes 1, Campus de Beaulieu, 35042 Rennes Cedex, FRANCE

E-mail address: hamadi.abidi@univ-renne1.fr

IRMAR, Université de Rennes 1, Campus de Beaulieu, 35042 Rennes Cedex, FRANCE

E-mail address: thmidi@univ-renne1.fr 